\title{
Immediate Non Invasive Aesthetic Management of Anterior Open Bite : A Case Report
}

\author{
Dr Kurien Varghese (Professor And HOD) ${ }^{1}$,Dr Roshni Abdul Vaheed \\ (Post Graduate Student),Dr Shamla Latheef (Post Graduate Student) ${ }^{2}$, \\ Department Of Prosthodontics, Azeezia College Of Dental Science And Research.
}

\begin{abstract}
Anterior open bite is considered as one of the most complex and difficult problems to treat in aesthetic dentistry. Over the years, various multidisciplinary treatment modalities involving orthodontic or surgical correction of anterior open bite have been successfully implemented. However, these options require multiple clinical visits as well as long term treatment duration.

This paper presents a case report of immediate non- invasive aesthetic management of anterior open bite using composite resin restoration.
\end{abstract}

Keywords: Anterior open bite, composite, aesthetic, time constraint.

\section{Introduction}

Anterior open bite arises due to various multi-factorial etiologies like skeletal abnormal growth pattern, dental, respiratory, neurologic, and habitual factors or tongue posture and function ${ }^{1-3}$.

Technological improvements and advancement in dental material science has made it possible to consider direct composite resin restorations as a viable alternative for patients that require anterior restorative procedures. In light of these improvements, interventions with composite resins have made possible the reestablishment of specific and individual details existing in the natural dentition, in a satisfactorily esthetic way and practically imperceptible to human vision. ${ }^{4}$ However, in order to achieve esthetic excellence, dentists should understand and apply artistic and scientific principles when choosing the shade of restorative materials, as well as during the application of the composite resin 5 .

\section{Case Report:}

A 19 year old male patient reported to the Department of Prosthodontics, Azeezia Dental College, with a complaint of anterior open bite (Figure 1) with edge to edge occlusal relation and wanted immediate correction. The patient was enlisted for admission with the Indian Naval Academy and had cleared all the preliminary tests. However, his anterior open bite was pointed out as a disqualifying factor by the medical officers in the Academy. He was given a duration of two days for possible correction of this defect before he appeared for his next medical fitness test.The ideal treatment plans, which would likely be orthodontic correction, occlusal equilibration or invasive procedures like intentional endodontic treatment followed by rehabilitation with crowns on all his maxillary and mandibular anterior teeth were not feasible options at this juncture considering his age, ethical concerns and the time constraints. So we had to opt for a non-invasive, aesthetic smile enhancing technique which could be completed in a single office visit using composite resin. The combination of the increasing demand of the patient for aesthetics and the capacity to preserve the dental structure resulted in applying this incremental technique for restoring his anterior open bite in the most non invasive and natural way. This article presents the utilisation of composite resin as a simple and effective tool for aesthetic reproduction of anatomic form of his anterior teeth to satisfactorily camouflage the present anterior open bite in this time bound situation.

\section{Procedure:}

1. Preliminary impressions of the maxillary and mandibular arches were made for diagnostic casts and treatment planning.

2. A wax mock up of the proposed restorations were done on the cast.

3. Using this as a guideline, a wax mock up was done intraorally to obtain the patient consent and acceptance before proceeding with the actual treatment.(Figure 2)

4. Putty index of the anterior wax mock up was made with Polyvinyl Siloxane impression material. (Flexceed, GC) (Figure 3)

5. This index was used as a template to place the composite (Te-Econom Plus, Ivoclar Vivadent) incrementally following an artistic approach bearing in mind the different nuances of each tooth which was being restored.

6. Isolation of the teeth and adjacent structures were maintained at all time during the treatment phase. 
7. Etching of the teeth was done following very minimal tooth preparation using the wax mock up and template as a guideline.

8. Bonding agent was applied and composite resin was placed incrementally following standard procedures.

9. Curing of composite resin was done using the composite curing unit ( 3 M ESPE, Elipar ${ }^{\mathrm{TM}}$ ) until function, form and aesthetics were adequately restored. (Figure 4)

10. Finishing and polishing of the restorations were performed after we were able to establish a reproduction of individual details existing in natural dentition in a satisfactorily aesthetic manner.(Figure 5)
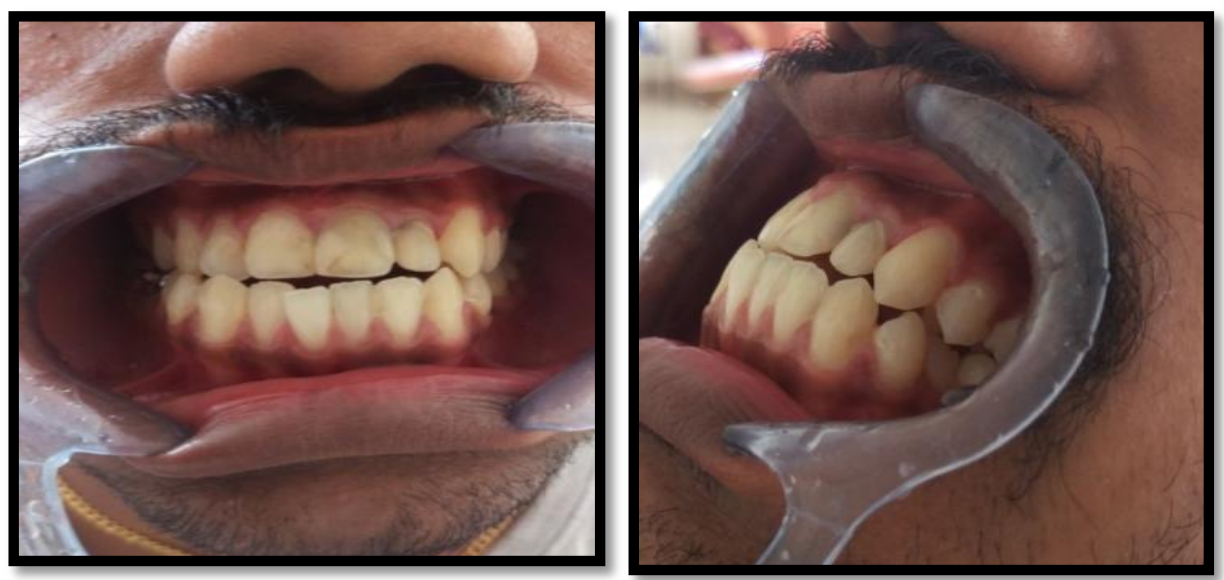

(Figure 1)
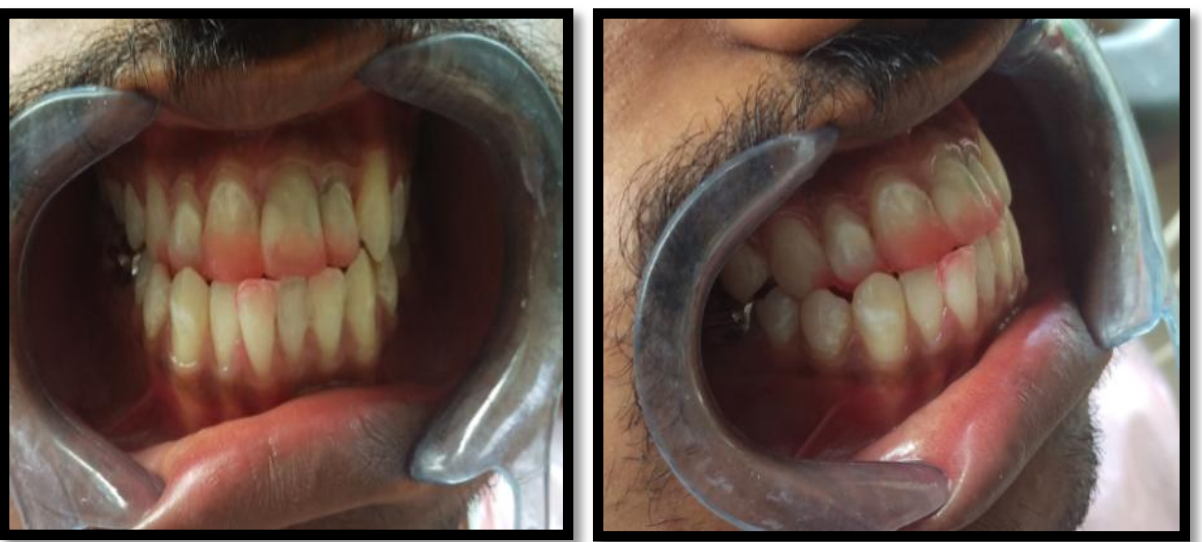

(Figure 2)

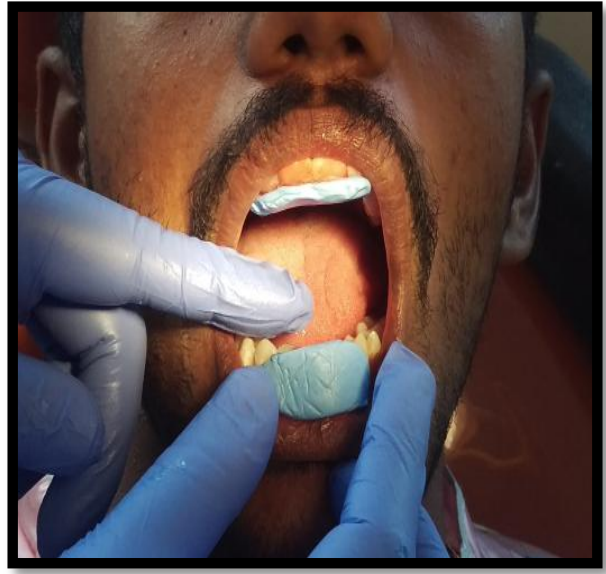

(Figure 3)

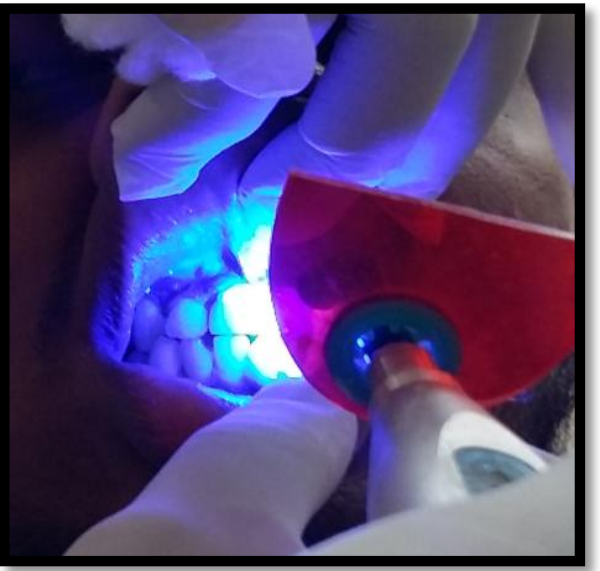

(Figure 4) 

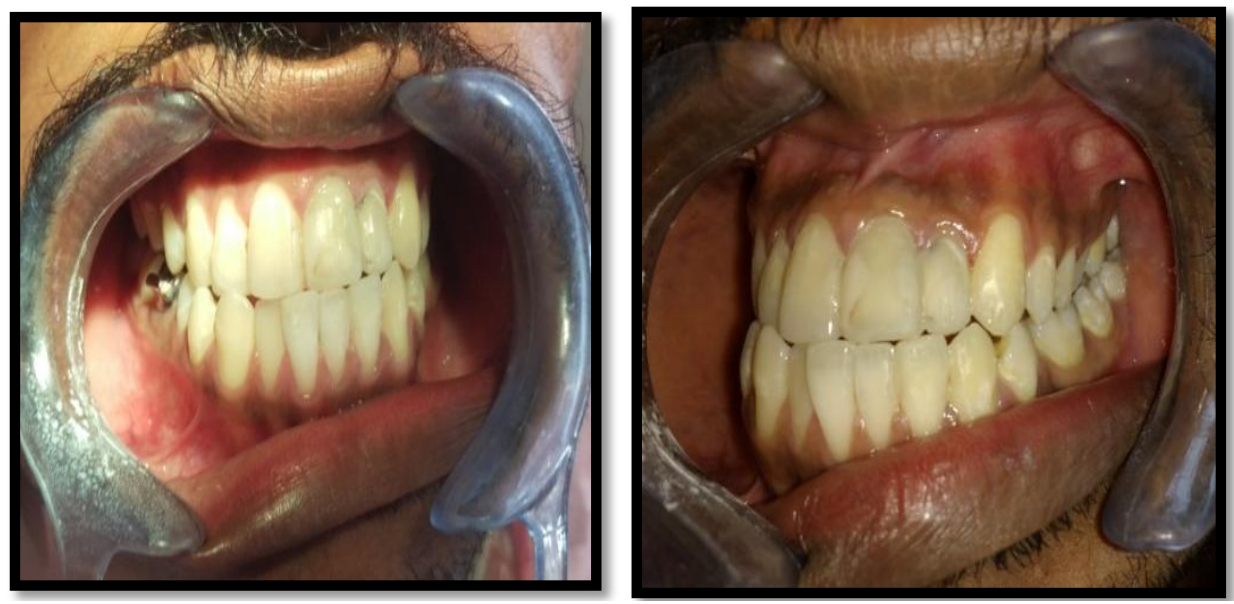

(Figure 5)

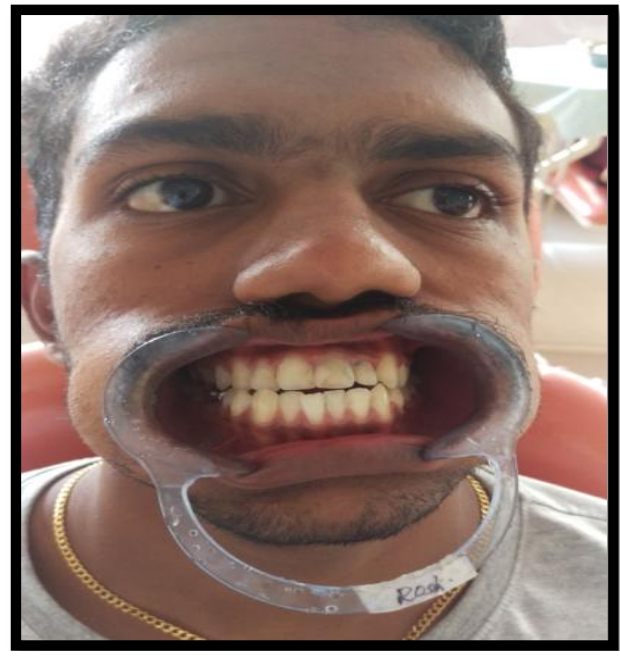

Pre-treatment

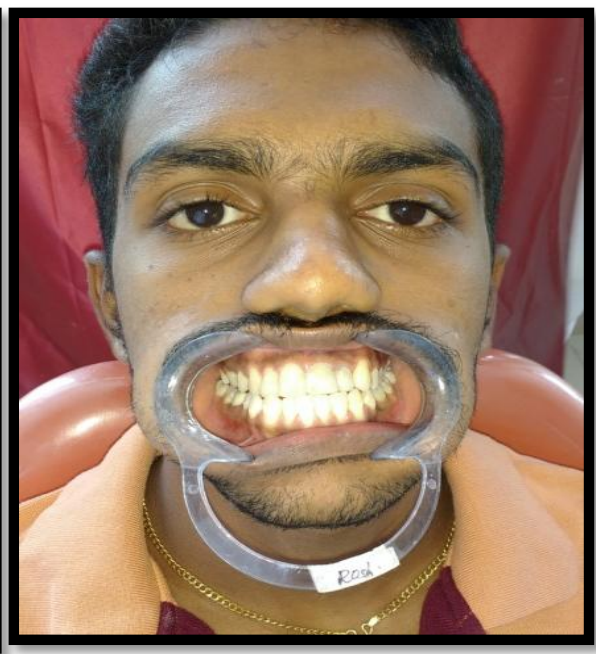

Post-treatment

\section{Conclusion}

This treatment approach of using direct procedures with composite resins was applied for restoring the anterior open bite due to the time constraints. This treatment plan aided in maintaining the aesthetics and at the same time preserved the dental structure so that we could opt for a permanent corrective treatment in due course of time.

\section{References}

[1]. Subtelny JD, Sakuda M. Open-bite: diagnosis and treatment Am J Orthod. 1964;50:337-358.

[2]. Burford D, Noar JH. The causes, diagnosis and treatment of anterior open bite. Dent Update. 2003;30:235-241.

[3]. Arat ZM, Isxeri H, Arman A. Differential diagnosis of skeletal open bite based on sagittal components of the face. World J Orthod. 2005;6: 41-50.

[4]. Araujo EM Jr, Baratieri LN, Monteiro S Jr, Vieira LC, Andrada MA. Direct adhesive restoration of anterior teeth: part 2. Clinical protocol. Pract Proced Aesthet Dent. 2003;15(5):351-7.

[5]. Robert Marus et al.Treatment planning and smile design using composite resin. pract Proced Aesthet Dent. 2006 May;18(4):23541.

[6]. Flavia Pardo Salata Nahsan, Rafael Francisco Lia Mondelli. Clinical strategies for esthetic excellence in anterior tooth restorations: Understanding colour and composite resin selection. J Appl oral Sci.2012 Mar-Apr;20(2); 151-156 